MATEC Web of Conferences 52, 01003 (2016)

DOI: $10.1051 /$ matecconf/20165201003

C) Owned by the authors, published by EDP Sciences, 2016

\title{
Circuit Modeling and EM Simulation Verification of DGS based Low-Pass Filter Employing Transmission Line Model along with Microstrip-Slotline Transitions
}

\author{
Mouloud CHALLAL ${ }^{1, a}$, Mokrane DEHMAS ${ }^{1}$, Arab AZRAR $^{1}$, Rabia AKSAS ${ }^{2}$ and Mohamed TRABELSI ${ }^{2}$ \\ ${ }^{1}$ Signals and Systems Laboratory, Institute of Electrical and Electronic Engineering, Dept. of Electronics, University \\ of Boumerdes, 35000 Boumerdes, Algeria \\ ${ }^{2}$ Electronics Department, Ecole Nationale Polytechnique, 16000 Algiers, Algeria
}

\begin{abstract}
In this paper, an equivalent circuit model (ECM) for a defected ground structure (DGS) pattern is proposed and evaluated for designing a compact low-pass filter (LPF). The proposed ECM is based on microstrip lines and microstrip-slotline transitions. Every slotlines of the DGS unit are modeled by ideals transmission lines of characteristic impedance and electrical length. Comparison between full-wave EM and circuit simulations illustrates the validity of the proposed ECM.
\end{abstract}

\section{Introduction}

Recently, various etched defects on metallic ground plane, namely, defected ground structure (DGS) have been focused by many researchers. They are very efficiently used to improve the performances of a variety of microwave circuits such as filters, amplifiers, oscillators and antennas [1]-[4]. Usually, a DGS unit in either microstrip or coplanar waveguide is modeled by lumped elements (LE), i.e, a parallel LC resonant circuit, known as conventional equivalent circuit model (CECM) connected to transmission lines at both sides [1]. Furthermore, some circuit models based on an inductor and a shorted stub for a spiral DGS and a short stub, additional series transmission lines and a resistor for a dumbbell DGS have also been presented in literature [5], [6]. Even though this type of circuit models can present a periodic frequency response, they require a full-wave electromagnetic (EM) simulation in order to determine the values of their elements. However, EM simulation takes a lot of time to accomplish the design goals. This is a first drawback of this modeling method. The second one is that there is not relationship between the circuit model parameters and the physical dimensions of the DGS. Consequently, this modeling technique is not practical for designing microwave circuits in general and filters in particular. To overcome these limitations, it is necessary to establish an accurate technique by developing another equivalent circuit model (ECM), which is derived from DGS physical dimensions and do not need to use EM simulation to achieve it parameters.

In this paper, a proposed equivalent circuit model (PECM) for the DGS is developed and a systematic method for the parameters extraction is described. Moreover, it is applied to the design a compact low-pass filter (LPF). The PECM is based on microstrip line model and microstrip-slotline transitions [7]-[9]. Every slotlines of the DGS are modeled by ideals transmission lines of

\footnotetext{
${ }^{\mathrm{a}}$ Corresponding author : mchallal@ieee.org
} 
characteristic impedance $\left(Z_{S i}\right)$ and electrical length $\left(\theta_{S i}\right)$, where i goes from 1 to $\mathrm{nb}$ (nb is the slotlines number). To show the validity of the PECM, three steps are taken in consideration and are as follows:

(i) Comparison between Full-wave EM, CECM and PECM simulations of the DGS unit;

(ii) Application of the PECM to design a compact LPF having three DGS units as an example of purpose;

(iii) Comparison between full-wave EM-LPF and PECM-LPF simulations.

The availability of substrate material, which is used in this work, is based on RO4350B Rogers material with a thickness of $0.25 \mathrm{~mm}$, conductor layers of $35 \mu \mathrm{m}$ and a relative permittivity of 3.63 .

\section{Equivalent Circuit Model}

Based on microstrip line model and microstrip-slotline transitions [7]-[9], a PECM is described in this section. As a first step, a dumbbell shaped DGS is chosen as an example of application that is shown in Figure 1.a. Next, the structure is modeled by a conventional parallel inductor (L) and capacitor (C) resonant circuit connected to transmission lines at both sides as shown in Figure 1.b, where the circuit elements are given by [1].

$$
\begin{aligned}
& C=\frac{\omega_{0}}{2 Z_{0}\left(\omega_{0}^{2}-\omega_{c}^{2}\right)} \\
& L=\frac{1}{\omega_{0}^{2} C}
\end{aligned}
$$

where $\omega_{0}=2 \pi f_{0}$ and $\omega_{c}=2 \pi f_{c}$ are respectively the angular resonant frequency and 3-dB cutoff frequency ( $f_{0}$ and $f_{c}$ are respectively the attenuation pole and cutoff frequencies, they obtained from the simulated response of a DGS unit using an EM simulator) of the DGS. $Z_{0}$ is the characteristic impedance of the transmission line.

The considered DGS unit is composed of two rectangular defected areas and a narrow connecting slot as shown in Figure 1.a. The dimensions a, b, g and c are considered to be respectively 5, 3.8, 0.5 and $1 \mathrm{~mm}$. Hence, the achieved attenuation pole and cut-off frequencies using an EM simulator are respectively $8 \mathrm{GHz}$ and $3.68 \mathrm{GHz}$. Insertion of these quantities into equations (1) and (2) yield values of $\mathrm{C}$ and $\mathrm{L}$ to be respectively $0.25 \mathrm{pF}$ and $1.57 \mathrm{nH}$. A comparison between CECM, PECM and fullwave EM simulations will be discussed later.
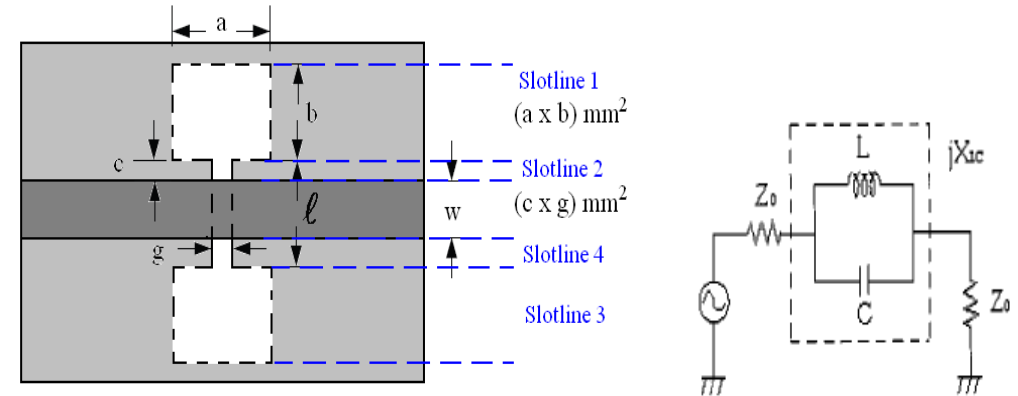

Figure 1. DGS unit. (a) Geometry of dumbbell shaped DGS and, (b) Its conventional equivalent circuit model (CECM)

The PECM for the DGS unit is shown in Figure 2 (Model 1 which corresponds to one DGS unit). The considered structure is modeled such that, all slotline are modeled by ideal transmission lines of characteristic impedance $\left(Z_{S i}\right)$ and electrical length $\left(\theta_{S i}\right)$, where $\mathrm{i}$ goes from 1 to $\mathrm{nb}$ (nb is the slotlines number) as shown in Figure 2. In our case, the Slotlines 1 and 3 and Slotlines 2 and 4 indicated in 
Figure 1.a are respectively the two rectangular defected areas (axb) and a narrow connecting slot (gxc). Next, the short-end of the slotline is represented by an equivalent length [9].

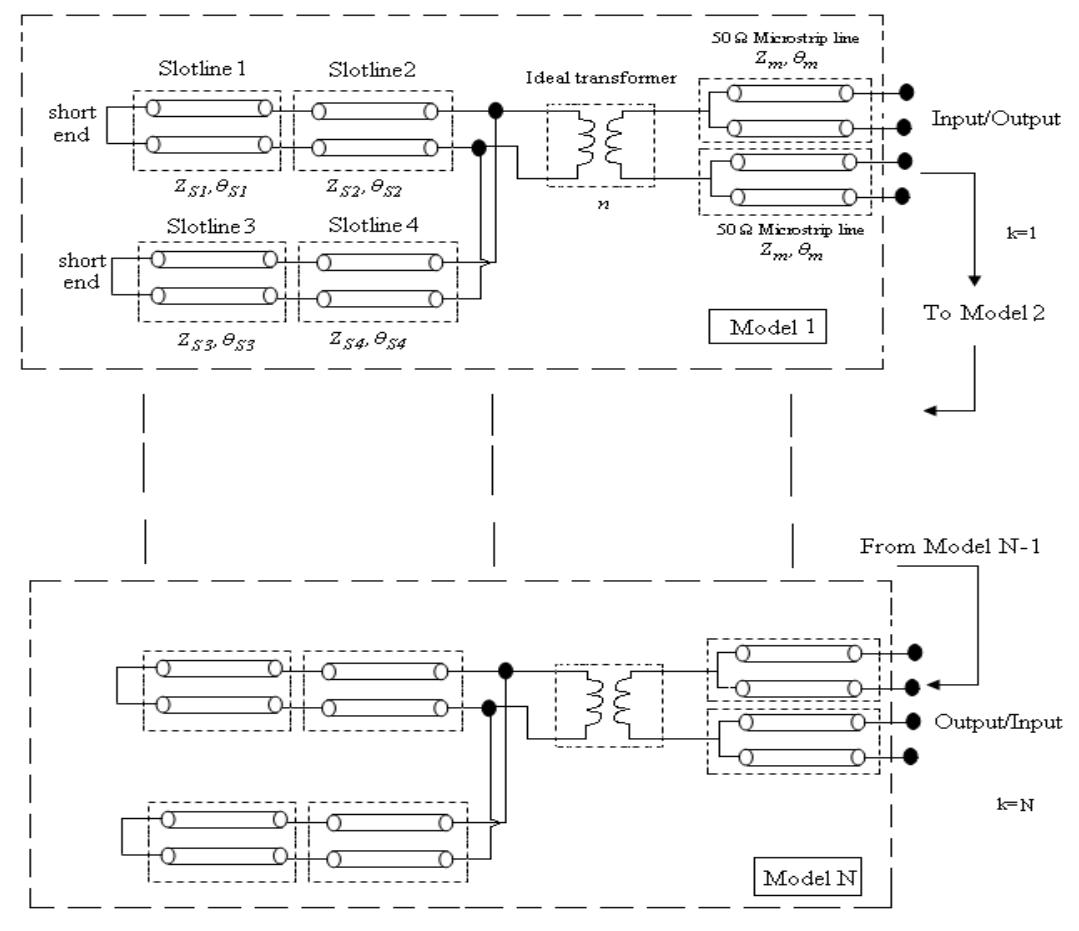

Figure 2. Proposed equivalent circuit model (PECM) for the cascaded defected ground structures (N: Number of used DGS patterns)

Finally, the transition of microstrip line to slotline, i.e, the EM coupling between them is modeled by an ideal transformer as shown in Figure 2, where $\mathrm{n}$ is the turn ratio of the transformer. In this work, for the simplification raison of calculus, the slotlines 1 and 2 are considered respectively similar to slotlines 3 and 4 as shown in Figure 1.a. The factor $k$ of Figure 2 is equal to the number of similar DGS patterns connected in cascade.

The slotline equations such as slotline wavelength and slotline impedance used in this work are the closed form expressions of Janaswamy and Schaubert [9] for low $\varepsilon$ r substrates obtained by curve fitting the numerical results achieved from Galerkin' $s$ method in the Fourier transform domain (FTD). Theoretical and measurements results of the short-end of the slotline obtained by Yang and Alexopoulos [10] and Knorr and Seanz [11], respectively, have expected that the end reactance of a shorted slotline is purely inductive and significant. It increases with the increase of both the slotline width and $h / \lambda_{0}$ ratio. The inductive reactance is expressed by an inductance $\left(L_{0 S}\right)$ connected to the end of a slotline. The equivalent length of the short-end effect $\left(\ell_{S 1_{-} e q}\right)$ may be up to $0.1 \lambda_{S 1}$ [9].

Using Agilent ADS simulator, the PECM is simulated for the purpose of comparison to the CECM and full-wave EM simulations. The schematic circuit of the PECM in ADS simulator is shown in Figure 3. The electrical lengths $l_{S I_{-} e q}, l_{S 1}$ and $l_{S 2}$ mentioned in Figure 3 correspond respectively to the electrical length of the short-end effect $\left(\theta_{S 1_{-} e q}\right), \theta_{S 1}$ and $\theta_{S 2}$. The values of $\theta_{S 1}$ and $\theta_{S 2}$ are selected by considering $f_{0}$ of $8 \mathrm{GHz}$ in. 


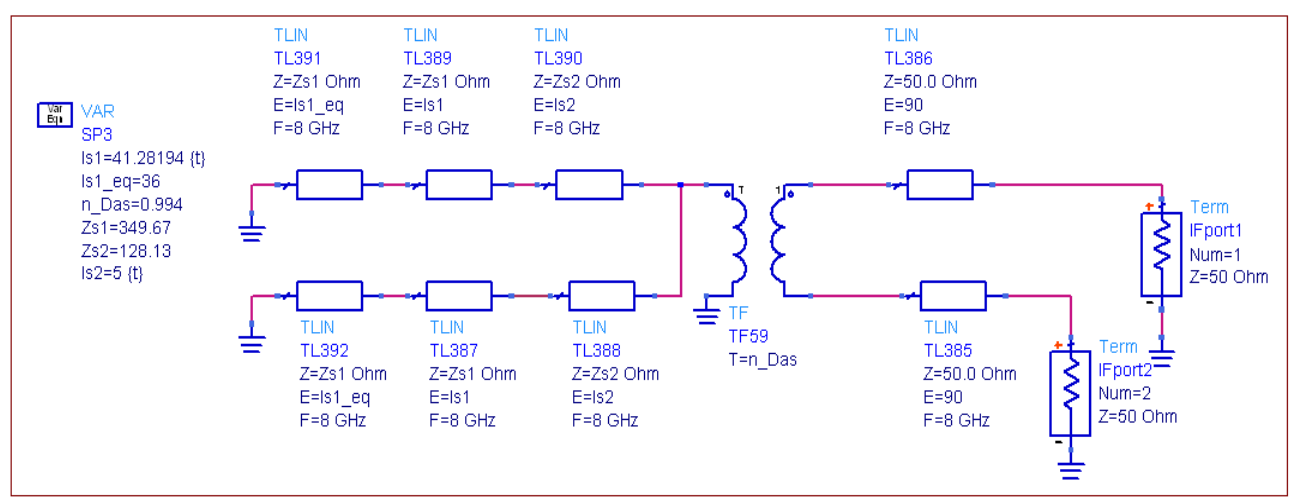

Figure 3. Schematic circuit of the PECM in ADS simulator

The required physical length for the first ideal transmission line including the equivalent length of the short-end effect $\left(L_{1}\right)$ as a function of both ratio $\lambda_{S 1} / \lambda_{0}$ (given in [9] for $0.075 \leq a / \lambda_{0} \leq 1$ ) and $f_{0}$ may be estimated by the following expression:

$$
\begin{aligned}
L_{1} & =L_{S 1}+\ell_{S 1_{-} e q} \approx 0.1145 \frac{c 10^{3}}{f_{0}} \frac{\lambda_{S 1}}{\lambda_{0}}+0.1 \lambda_{S 1} \\
& \approx 343.5 \frac{10^{8}}{f_{0}} \frac{\lambda_{S 1}}{\lambda_{0}}+0.1 \lambda_{S 1}
\end{aligned}
$$

where $L_{S 1}$ is the physical length of the first ideal transmission without the equivalent length of the short-end. The frequency $f_{0}$ is given in GHz.

Similarly to the estimated $L_{1}$, the required physical length for the second ideal transmission line as a function of both ratio $\lambda_{S 2} / \lambda_{0}$ (given in [9] for $0.0015 \leq \mathrm{g} / \lambda_{0} \leq 0.075$ ) and $f_{0}$ may be estimated by the following expression:

$$
L_{2} \approx 0.0137 \frac{c 10^{3}}{f_{0}} \frac{\lambda_{S 2}}{\lambda_{0}} \approx 41.1 \frac{10^{8}}{f_{0}} \frac{\lambda_{S 2}}{\lambda_{0}}
$$

Figure 4 shows comparisons of EM and circuit models simulations. In Figure 4, it is observed that an excellent agreement has been found between Full-wave EM and PECM simulations. This result shows that the PECM provides excellent performances and gives results comparable to the conventional one.

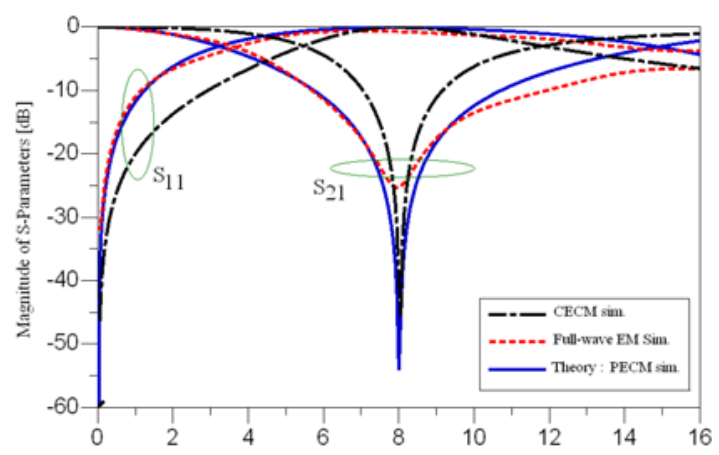

Figure 4. Magnitude of $\mathrm{S}_{21}$ and $\mathrm{S}_{11}$ of DGS unit, CECM, PECM and Full-wave EM simulations 


\section{Application of the proposed equivalent circuit model to design a microwave LPF with DGS}

In order to show the validity of the PECM, a DGS-dumbbell LPF has been designed and simulated. To improve the transfer function response $\left(\mathrm{S}_{21}\right)$ of the DGS unit shown in Figure 4, full-wave EM and circuit simulations with increasing number of cascaded dumbbell-shaped DGS units are performed. It has been observed that a good response is achieved when three or more units are cascaded. Figure 5 shows the layout of three units of DGS-LPF whereas Figure 6 shows the magnitude of $S_{21}$ and $S_{11}$ obtained by both full-wave EM and PECM simulations.

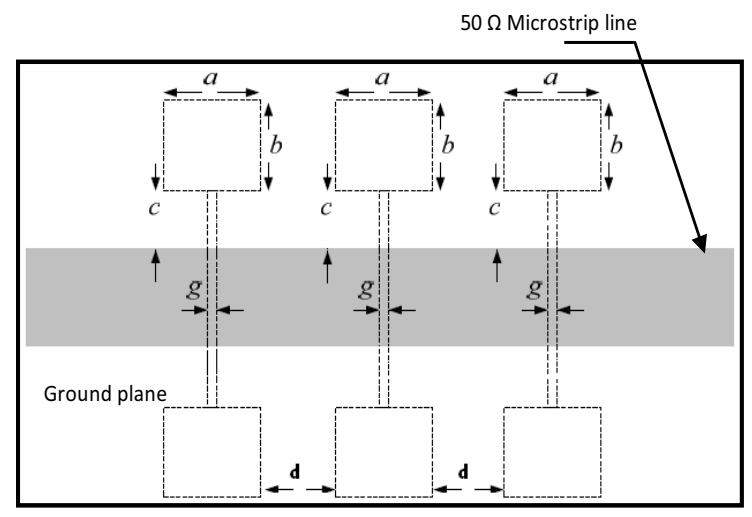

Figure 5. Layout of LPF based on three DGS slots
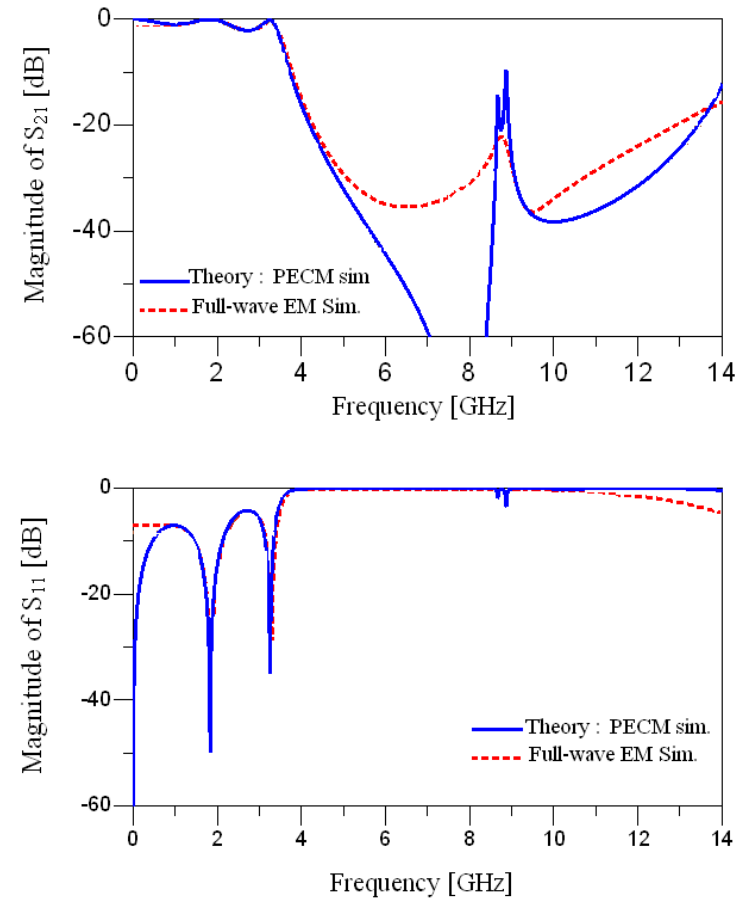

Figure 6. Magnitude responses of the DGS-LPF : Theory (PECM simulation) and full-wave EM simulation

From Figure 6, it can be seen that the simulation results obtained by the PECM (theoretical) agree well with full-wave EM-simulations results. Besides, less time is consumed when simulations are 
performed using the PECM. Consequently, when the number of DGS in a circuit is large (eg. more than two DGSs), the simulations using the PECM is more advantageous than the full-wave EM simulations.

\section{Conclusion}

An Equivalent circuit model (ECM) using transmission line model along with microstrip-slotline transitions has been proposed to illustrate and design defected ground structure (DGS) unit. All slotlines of the DGS unit have been modeled by ideals transmission lines of characteristic impedance Zs and electrical length $\theta_{\mathrm{S}}$. Also, The EM coupling between microstrip line and slotline is modeled by an ideal transformer. To qualify the proposed circuit model, a microwave LPF with DGS based on the proposed ECM is designed. A good agreement between the circuit simulation and full-wave EMsimulations of the filter characteristic validates the proposed ECM of the microwave DGS based LPF.

\section{Acknowledgment}

The authors express their thanks to Dr. Khaled Ben Ali, University catholique de Louvain, Belgium, for providing support and assistance to perform simulations by using software.

\section{References}

1. F. Wei, X. Shi, B. Li, Q. Huang and X. Wang, Micowave and optical technology letters, 50, (2008).

2. A. Boutejdar, M. Challal and A. Azrar, The Applied Comp. Electromagnetics Society, 26, (2011)

3. Y. T. Lee, J.S. Lim, J.S. Park, D. Sangwook Nam, IEEE Microwave and Guided Wave Letters, 12, 2002.

4. M. Challal, A. Azrar and M. Dehmas,, IJCSI- Int. J. of Comp. Science Issues, 8, ( 2011).

5. C. S. Kim, J. S. Lim, S. Nam, K. Y. Kang and A. Ahn, Electron. Lett., 38, (2002).

6. J. Park, K. Park, S. Chang and D. Ahn, Proc. 4th International Conference on Electronics, Hardware, Wireless and Optical Communications, (2005)

7. J. B. Knorr, IEEE Trans. Microwave Theory Tech., (1974)

8. B. Schuppert, IEEE Trans. Microw. Theory Tech., 36, (1988)

9. K. Gupta, R. Garg, I. Bahl, and P. Bhartia, 2nd ed, Artech House, (1996)

10. H. Y. Yang and N. G. Alexopoulos, IEEE Trans. Microwave Theory Tech., 36, (1988)

11. J. B. Knorr and J. Seanz, IEEE Trans. Microwave Theory Tech., 21, (1973) 